\title{
Sleep Inertia Countermeasures in Automated Driving: A Concept of Cognitive Stimulation
}

\author{
Johanna Wörle $^{1, *}$, Ramona Kenntner-Mabiala ${ }^{1}$, Barbara Metz ${ }^{1}$, Samantha Fritzsch ${ }^{1}$, \\ Christian Purucker ${ }^{1}$, Dennis Befelein ${ }^{1}$ and Andy Prill ${ }^{2}$ \\ 1 Würzburg Institute for Traffic Sciences; 97209 Veitshöchheim, Germany; kenntner@wivw.de (R.K.-M.); \\ metz@wivw.de (B.M.); fritzsch@wivw.de (S.F.); purucker@wivw.de (C.P.); befelein@wivw.de (D.B.) \\ 2 Hyundai Motor Europe Technical Center GmbH; 60547 Rüsselsheim, Germany; \\ amprill@hyundai-europe.com \\ * Correspondence: woerle@wivw.de; Tel.: +49-931-78009-124
}

Received: 26 May 2020; Accepted: 28 June 2020; Published: 30 June 2020

\begin{abstract}
When highly automated driving is realized, the role of the driver will change dramatically Drivers will even be able to sleep during the drive. However, when awaking from sleep, drivers often experience sleep inertia, meaning they are feeling groggy and are impaired in their driving performance-which can be an issue with the concept of dual-mode vehicles that allow both manual and automated driving. Proactive methods to avoid sleep inertia like the widely applied 'NASA nap' are not immediately practicable in automated driving. Therefore, a reactive countermeasure, the sleep inertia counter-procedure for drivers (SICD), has been developed with the aim to activate and motivate the driver as well as to measure the driver's alertness level. The SICD is evaluated in a study with $\mathrm{N}=21$ drivers in a level highly automation driving simulator. The SICD was able to activate the driver after sleep and was perceived as "assisting" by the drivers. It was not capable of measuring the driver's alertness level. The interpretation of the findings is limited due to a lack of a comparative baseline condition. Future research is needed on direct comparisons of different countermeasures to sleep inertia that are effective and accepted by drivers.
\end{abstract}

Keywords: highly automated driving; sleep; sleep inertia; HMI design

\section{Introduction}

Highly automated driving systems (ADS) are about to be introduced to the market and they have the potential to change the way we travel fundamentally. Technologies such as Internet of Things, Big Data and Connected Vehicles further promote the progress in the development of ADS [1]. Surveys that were conducted on user requirements with regard to ADS reveal how potential users want to spend the gained time that they do not have to spend on controlling the vehicle. Among the most desired activities are phoning, mailing, interacting with passengers, eating and drinking, watching movies and resting [2]. In a more recent survey in five countries, "sleeping and relaxing" was stated as the preferred way to spend an automated drive [3]. This desire can be explained by the requirements of the modern lifestyle with long working hours and extended time spent on commuting. Some $30 \%$ of U.S. employees report sleeping less than six hours per night [4]. Thus, the option to use the commute to work for a nap appears highly promising for drivers.

Those ADS that are currently on the market (such as Tesla Autopilot, General Motors Super Cruise, or Mercedes-Benz Distronic Plus) do not offer the option for the driver to sleep. They are partially automated (level 2 according to the taxonomy of the Society of Automobile Engineers, SAE [5]) and therefore have to be supervised by the driver at all times. However, the ADS technology is advancing fast, and when reaching the level of high automation (SAE level 4), sleep can be implemented as a 
use case during the automated drive. The concept of "dual mode vehicles" in level 4 automation includes the option for a driver to engage in manual driving or the ability of a driver to take over in situations the system cannot handle [5]. Hence, questions arise on the impact of driver state after sleep. After awakening from sleep, humans experience a "period of transitory hypovigilance, confusion, disorientation of behavior and impaired cognitive and sensory-motor performance" ([6], p. 834), called "sleep inertia".

Sleep inertia is widely recognized and well-regulated in operational domains. In aviation, e.g., where pilots are allowed to take a nap during a flight, standardized nap protocols are in place to avoid performance impairment due to sleep inertia. Pilots on long-haul flights are allowed and even advised to sleep to restore their alertness throughout the flight. In order to avoid performance decrements after sleep, and thus potential safety risks, a procedure called the "NASA nap" is implemented. The NASA nap is a standardized rest period of $40 \mathrm{~min}$ with the opportunity to sleep followed by a 20-min period of wakefulness to overcome sleep inertia before returning to duty [7]. The duration of sleep is restricted to avoid deep sleep which produces the highest magnitude of sleep inertia. The NASA nap is recommended with slight differences in various aviation operator guidelines $[8,9]$.

In the AD domain, there are no guidelines and no common understanding on how to deal with a sleeping driver. The first driving simulator studies on human performance after sleep indicate that after sleep, drivers are impaired in their ability to engage in vehicle control and their driving performance is worsened $[10,11]$. Adverse driver states are a major safety issue in conventional driving to date. In automated driving, the safety impact of an adverse driver state is especially crucial in take-over situations, i.e., in the period after taking back vehicle control from automated to manual driving. EuroNCAP, the European car safety assessment program, introduces reliable driver state monitoring and effective action when an adverse driver state is detected as a primary safety measure [12]. This could mean that, in the case of the driver monitoring system detecting a driver getting too drowsy, it will warn the driver or even initiate a safety manoeuver. Drivers who awaken from sleep experience critical impairments in their take-over and driving performance [10]. The duration of sleep inertia depends on various factors such as the duration of prior sleep or the sleep stage the driver is awakened from [13]. It is thus critical to assess the driver's readiness to engage in vehicle control after sleep to avoid safety-critical situations. The second approach is to actively counter sleep inertia and thus performance impairment and reduce the severity and duration of impaired performance after awakening.

The aim of the paper is to make a proposal of a reactive strategy to deal with sleep inertia in AD and start a discussion on sleep inertia countermeasures in this new field of application.

\subsection{Sleep and Sleep Inertia}

Sleep is broadly defined as a "reversible behavioral state of perceptual disengagement from and unresponsiveness to the environment" [14] (p. 15). Sleep itself is not a constant state but rather characterized by an alternation of different sleep stages. The sleep stages according to the American Academy of Sleep Medicine (AASM) standard [15] are:

- W: Wakefulness

- N1: Light sleep or dozing

- N2: Stable sleep

- N3: Deep sleep or slow wave sleep

- $\quad$ R: REM-sleep or dream sleep

The transitional phase from sleep to wakefulness is also a distinct state characterized by "hypovigilance, confusion, disorientation of behavior and impaired cognitive and sensory-motor performance" ([6], p. 834), called "sleep inertia". Physiologically, the state of sleep inertia is characterized by a decreased cerebral blood flow [16]. Spectral analyses of the EEG show higher power in the delta-theta and alpha frequency range and a lower power in the beta frequency range which 
indicates low general alertness [17,18]. Hilditch and McHill [19] suggest that the function of sleep inertia might be for the organism to promote sleep upon awakening so that sleep can be maintained when the awakening is undesired.

In the post-awakening period, performance impairment is evident in a wide range of tasks. Most laboratory studies investigate human performance after sleep with highly standardized tasks: the Psychomotor Vigilance Task (PVT) is widely used in studies on fatigue, but also on sleep inertia [20-22]. The PVT is a standardized test that measures alertness. Subjects have to respond to a visual stimulus as quickly as possible. One of the advantages of the PVT is that it has no learning curve. After sleep, subjects react slower to the stimuli [21,22] and they have more lapses [20]. Other studies assessed the working memory upon awakening: In the n-back task, a subject is presented with a sequence of stimuli and they have to react when the current stimulus matches the one from $\mathrm{n}$ steps earlier. Groeger and colleagues [23] applied a 1-, 2- and 3-back task to investigate impairments in working memory on tasks of rising difficulty after 90 -min naps. They found stronger performance decrements on tasks which highly rely on executive functions. The Digit Symbol Substitution Test (DSST) assesses working memory and processing speed by presenting digit-symbol pairs followed by a list of digits. Subjects have to assign the correct symbol as fast as possible. The number of correct responses was lower after sleep than before [22,24].

The magnitude and duration of sleep inertia is shaped by many factors. A circadian influence seems apparent with sleep inertia being stronger in the circadian low i.e., during the biological night $[21,25,26]$. An important factor that influences the magnitude of sleep inertia is the sleep stage prior to awakening. Deep sleep (or slow wave sleep, SWS) produces the highest impairments due to sleep inertia [25,27]. For other sleep stages, results are ambiguous: Cavallero and Versace [28] found a higher impairment of performance on a reaction time task after N2 sleep than after REM sleep. Reaction times were prolonged after N2 sleep compared to N1 sleep [29]. Scheer and colleagues [21] found no differences in the performance of an addition task between subjects awakening from N2, deep sleep or REM sleep. The duration of sleep inertia ranges from $1 \mathrm{~min}$ up to $4 \mathrm{~h}$ depending on the study design. However, without major sleep deprivation, a duration of more than $30 \mathrm{~min}$ is unlikely [13].

\subsection{Countering Sleep Inertia}

Sleep inertia can be a serious safety issue especially in settings where optimal human performance is crucial under adverse conditions. Such conditions can be extended working hours, working during the circadian trough or traveling through different time zones. In those operational domains, Fatigue Risk Management Systems (FMRS) are in place to avoid safety risks due to impaired alertness, e.g., fatigue or sleep inertia. Different strategies can be distinguished to counter sleep inertia: proactive strategies are commonly recommended in work guidelines that regulate, e.g., sleep schedules or nap durations to avoid sleep inertia. Reactive countermeasures are implemented after awakening when sleep inertia is already present.

Proactive strategies to mitigate sleep inertia are well-established in operator guidelines or shift schedules, e.g., in hospitals or the transportation industry. They include recommendations on sleep schedules, avoid awakening during the circadian low and strategic naps. A common proactive strategy to minimize sleep inertia is the 'NASA nap'. The total sleep duration is restricted to avoid deep sleep, and after awakening, operators have to wait for $20 \mathrm{~min}$ to return to duty to overcome sleep inertia. It has to be mentioned, however, that the 40-min rest opportunity of the NASA nap does not fully avoid deep sleep. Even in the original study on the NASA nap, some pilots entered deep sleep within this short period [7]. Since deep sleep occurs in cycles throughout the sleep period, awakening after 80-100 min could be an alternative. After this time the first whole sleep cycle with the first deep sleep period is normally finished [13]. It was found, for instance, that sleep inertia magnitude was greater after a 40 min nap than after a 60 min nap [30]. Ferrara and De Gennaro [6] suggest that awakenings after extended periods of sleep deprivation and during the circadian trough (i.e., during the night) 
should be avoided. The implementation of proactive strategies is that they require a planned sleep opportunity and a planned wake-up time.

Reactive countermeasures are not commonly implemented and empirical evidence on their effectiveness is incomplete. Examples for applications of reactive countermeasures are light alarms that claim to wake the user more gently and thus minimize sleep inertia. Hilditch, Dorrian and Banks [31] give an overview of the literature on reactive sleep inertia countermeasures. The review includes studies on caffeine, light (postwaking), light (prewaking), sound, temperature, self-awakening and face-washing. Studies included in the review assess the impact of these countermeasures on either subjective alertness or objective alertness (i.e., physiology or performance) or both. One main conclusion of the authors is that there is a gap in the evidence-base of research on sleep inertia countermeasures. Caffeine administered before sleep is suggested as the most effective reactive sleep inertia countermeasure. Empirical evidence on the effectiveness of light or temperature is not sufficient to draw conclusions at this point.

\subsection{Implications of Sleep Inertia in Automated Driving}

Driving automation has not yet progressed to a level that allows drivers to sleep during the drive. Current ADS require the driver to supervise the ADS at all times. Despite that, videos are making the headlines that show drivers sleeping behind the wheel of their automated vehicles [32,33]. At the current stage, sleep is a clear misuse and has to be avoided at any cost. However, with progress in the development of ADS, the systems will be able to execute all parts of the driving task reliably within the system boundaries. Fully automated driving is not realized throughout all road sections of a trip. That is why at some point of the drive (e.g., at a motorway exit, or when boarding a ferry) the user will be required to execute the driving task manually. The concept of dual-mode vehicles outlined by the SAE [5] explicitly refers to this design option where the user of a highly ADS has the option to request manual driving if she or he wants. The user can therefore switch actively between a user state and a driver state. Users therefore will be allowed to sleep but when they take back the driving task as a driver, it has to be ensured that they are fit to drive after awakening. An exemplary use case could be a business trip where a saleswoman starts her drive with a highly automated vehicle early in the morning. The trip consists of two hours of motorway driving and after leaving the motorway, a rural road leads to her destination. The ADS only supports driving on the motorway, but not on rural roads. The saleswoman uses the motorway section to get some more sleep and is alerted by the ADS before the motorway exit. This way she is able to take back vehicle control before entering the rural road. The ADS ensures that after awakening, the driver's manual driving ability is not impaired due to sleep inertia.

When humans awake from sleep, they experience sleep inertia and are therefore impaired in their ability to drive $[10,11]$. Sleep inertia as a driver state has barely been an issue in road transport research so far. A study of take-over performance after sleep yielded clearly impaired performance when drivers take control back from the ADS [10]. Drivers' take-over reactions (i.e., glance at the road, glance at the mirrors, hands on the wheel) were all delayed by a few seconds after sleep compared to an awake baseline. It also seemed that the ADS's HMI display was a more important source of information. After sleep, drivers first checked the information on the HMI display before taking over. In contrast to that, the drivers ignored it when taking over after wakefulness. Most importantly, the drivers' overall performance in the take-over situation was worse after sleep. Drivers' lane keeping was clearly impaired and they performed fewer safety glances when changing lanes. Drivers subjectively perceived the take-over situations as more critical after sleep. In another driving simulator study, the focus was on the drivers' manual driving behavior during the first $10 \mathrm{~min}$ after sleep [11]. After being awakened by a request to intervene, drivers had to drive manually on a monotonous motorway for about $10 \mathrm{~min}$. Lane-keeping performance was clearly impaired after sleep. This effect was mainly evident in the first two minutes of the manual drive. After sleep, drivers drove at a reduced speed and they had problems 
keeping to a constant speed. The speed-keeping performance did not improve significantly in the course of the 10-min drive.

The presented findings from previous studies emphasize the necessity for a framework to minimize sleep inertia and associated safety risks in automated driving. Established strategies from other operational domains might only be partly transferable to AD. One approach could be-similar to the NASA nap — to limit rest periods of the driver during the drive, in order to avoid deep sleep. However, this might hardly be acceptable for the driver. If, for example, a two hour period of uninterrupted $\mathrm{AD}$ is available, it might not be communicable to the driver that they are only allowed to rest for $40 \mathrm{~min}$. Another strategy could be to awaken the driver early enough to let sleep inertia dissipate before they re-engage in driving. In this case however, the ADS has to ensure that the driver does not go back to sleep in the meantime. Proactive approaches to deal with sleep inertia are usually designed for professionals like pilots or hospital workers that are trained in alertness management. It cannot be expected from regular drivers to stick to such protocols. Therefore, we argue that technical solutions are to be preferred in AD.

Instead of proactive strategies to avoid sleep inertia, reactive strategies that counteract it seem highly promising in AD. While proactive strategies are thoroughly investigated and implemented, e.g., in industry guidelines, there is not much research on strategies to minimize sleep inertia after awakening. Some authors [6] suggest that sleep inertia can be reduced by stimulating or activating the individual after sleep. Everyday strategies like washing one's face with cold water or intensive stretching is not applicable in the vehicle cabin (although one could think of physical activities that could be performed while seated, similar to exercises suggested by some airlines for on-board fitness).

Due to the mainly cognitive requirements of the task of vehicle control, a more promising approach is to cognitively stimulate the driver after sleep. A very popular approach in daily life is a task-based mobile alarm app for smartphones. The basic principle is, that after awaking from sleep, one has to complete a task on their smartphone to ensure that they wake up reliably. Examples for tasks are taking a picture or solving math problems [34]. The activation through cognitive stimulation can promote cerebral activity on the one hand and be motivating because of its playful character on the other. However, there is barely any empirical evidence for the effectiveness of such task-based alarms.

Besides cognitive stimulation and motivation of the driver, our concept had a third aim: similar to established measurements of alertness such as the PVT, we aimed at assessing the alertness level of the driver after waking to assess the driver's fitness to drive.

A prototype sleep inertia countermeasure was developed and is tested in a driving simulator setup to evaluate its effectiveness in terms of:

- cognitively and physiologically activating the driver after sleep.

- motivating the driver after sleep.

- $\quad$ assessing the driver's alertness level to determine their readiness to drive.

\section{Materials and Methods}

\subsection{The Concept of the Sleep-Inertia Counter-Procedure for Drivers}

Two expert workshops were conducted with the aim to work out prototype wake-up concepts and a framework for a concept to counter sleep inertia in automated driving. $\mathrm{N}=8$ and $\mathrm{N}=9$ experts with backgrounds in human factors, traffic psychology and HMI design participated in the workshops. After several iterations, a concept for a sleep inertia counter-procedure for drivers (SICD) was developed and implemented as a tablet application in the driving simulation.

The basic idea of the SICD was to minimize sleep inertia by activating the driver after sleep as it is suggested by [6]. Suggestions such as washing one's face with cold water or physical exercise were discussed but rejected since they were not practicable in a vehicle cabin. Other reliable methods to counter sleep inertia such as caffeine administration [20] were rejected because they were judged to be too intrusive. One approach that was assessed to be feasible as an HMI solution was to cognitively 
activate the driver with a challenging task. Another advantage of a cognitive task is that it can also be used as a diagnostic tool to measure the alertness level of drivers after sleep similar to, e.g., the PVT. The SICD was designed with a gamification approach so that it was perceived as motivating and created a positive feeling.

The SICD was implemented as a gaming application on a tablet similar to a classical choice-reaction task, see Figure 1. Purple and turquoise dots appeared on a tablet screen every 1-2 $\mathrm{s}$ at random positions of the play area. Both the position of appearance and the time point were defined by random number generators. Frequency and position varied to avoid predictability and thus repetitive behavior and boredom. Drivers had to hit all target stimuli (purple dots) and avoid distractor stimuli (turquoise dots). To promote drivers' motivation during the task they received motivating messages such as "You are doing great". The duration of the SICD was $10 \mathrm{~min}$.
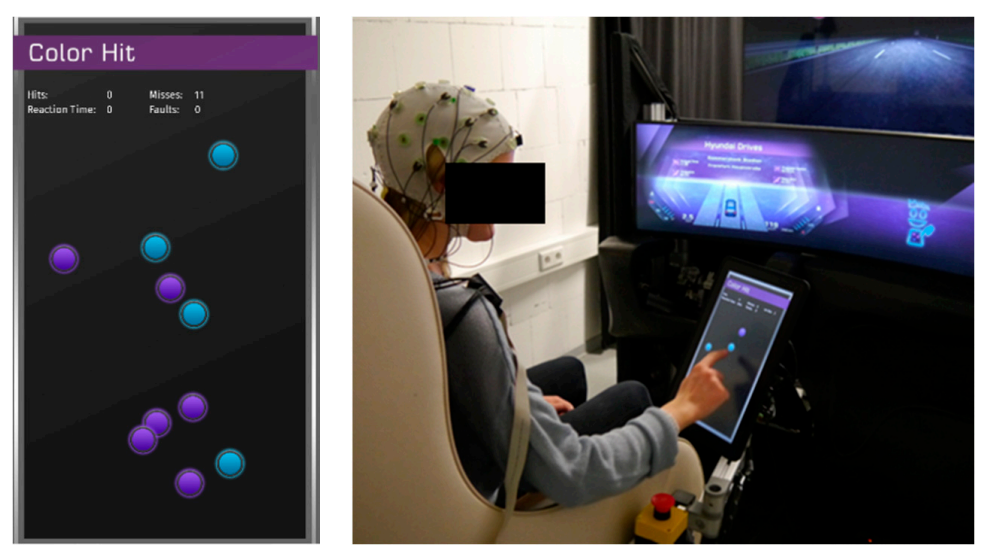

Figure 1. Screenshot of the sleep inertia counter-procedure for drivers (SICD, left) and participant equipped with EEG electrodes performing the SICD (right).

If participants hit a purple target dot, the counter registered a "hit". For all hits, reaction times were recorded. If participants hit a turquoise dot, a "fail" was counted. If a target dot was not hit, a "miss" was counted. Reaction times were calculated starting with the appearance of the dot on the screen until it was hit. The four parameters hits, fails, misses and reaction times were supposed to serve as measures for alertness, similar to established alertness measurements, such as the PVT.

\subsection{An L4 Concept Driving Simulator to Investigate Sleep}

The evaluation study was conducted in a driving simulator using the simulation software SILAB. The simulator was specifically designed for evaluating HMI concepts for automated driving. The main components were a dashboard with a steering wheel and a large diagonal display. Accelerator and brake pedal were available in manual driving mode. The driver was seated in a comfortable seat with a central infotainment touch display. All relevant components of the driving simulator were equipped with electric linear actuators and could be controlled via a computer. It was possible to move the seat to a lying position and to retract the steering wheel and pedals so that the driver had more space. Therefore, the cabin concept in the "manual driving mode" was different with the seat in the upright position and steering wheel and pedals extended while in the "automated driving mode" the seat was moved backwards and steering wheel and pedals were retracted and in "sleep mode" the seat was moved to a horizontal position.

Two different wake-up procedures were developed and implemented. The first wake-up procedure focused on a reliable awakening with a loud and sharp sound and flashing lights. The second wake-up procedure focused on a comfortable and pleasant wake up with soft music and a warm yellow light concept. The two wake-up procedures were tested between-subjects in the driving simulator study. However, no results will be presented on the acceptance and effectiveness of the wake-up procedures since this is outside the scope of this paper. 


\subsection{Study Design}

The study was conducted at the premises of the Würzburg Institute for Traffic Sciences (WIVW). $\mathrm{N}=21$ test participants ( 10 female, mean $_{\text {age }}=33, \mathrm{sd}=8$ ) completed two driving sessions in an L4 driving simulator using a highly automated driving system. All participants were recruited from the WIVW driver panel. Session 1 was scheduled during the daytime and session 2 was scheduled at 6 a.m. after a night of partial sleep deprivation, i.e., drivers were allowed to sleep no more than 4 $\mathrm{h}$. The aim was to get the drivers to fall asleep in the driving simulator. Each session started with a prequestionnaire and ended with a postquestionnaire.

In session 1, drivers first gave their informed consent, filled in the prequestionnaire and were then familiarized with the driving simulator, i.e., they learned the system handling and drove manually for $10 \mathrm{~min}$. Then they practiced the SICD. After the familiarization, the test drive started. For a graphical representation of the test drive, see Figure 2. The test drive started on a parking lot and drivers entered the highway. On the highway they activated the automated mode, then the vehicle drove automatically and the vehicle cabin also changed: the steering wheel folded back, the wide screen moved in near to the participant and the driving seat moved backwards and tilted back slightly so that the driver was in a more comfortable position. Then, the system offered the sleep mode that the driver confirmed with a button press. The screen turned darker and the driver's seat tilted to a lying position. Then, drivers were instructed to close their eyes and relax but not to sleep. After two minutes, drivers were alerted with either of two "wake-up procedures". Then they were asked to rate their subjective arousal and their subjective well-being on a slightly adapted version of the 9-point Self-Assessment Manikin (SAM) scale [35]. The SAM-scale is a "non-verbal pictorial assessment technique" ([35], p. 49). For our purposes, the valence scale showed five manikins displaying a scale ranging from an unhappy face expression to a happy face expression. Participants were asked "How good do you feel?" On the arousal scale the manikins ranged from a relaxed looking manikin with closed eyes to a very active manikin. Participants were asked "How activated are you?"
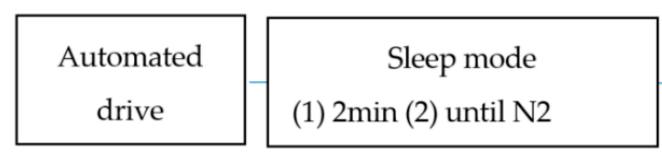

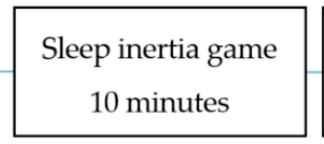

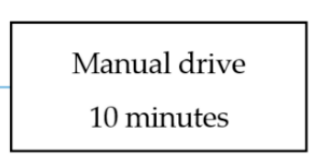

Figure 2. Schematic sequence of the test drive.

After the rating, the SICD was offered on the tablet screen and was started by the drivers via button press. The SICD was executed for $10 \mathrm{~min}$ and when it was finished, drivers were asked again to rate their well-being and their arousal. Then they performed a 10-min manual drive on a 3-lane highway with low traffic volume. After the manual drive, drivers rated their subjective well-being and arousal.

Session 2 had a similar procedure with the only differences that it took place at 6 a.m. and drivers were sleep deprived. Participants arrived at the test facilities by taxi and after filling in the pre-questionnaire, they were equipped with the EEG. Electrodes were placed according to the International 10-20 system [36]. The procedure of the test drive was the same as in session 1, but drivers were awakened when a sleep expert confirmed sleep stage N2 via EEG evaluation. Sleep stage N2 was chosen since it is the "deepest" stage that is considered appropriate during a nap in most operational guidelines. After awakening, drivers engaged in the SICD for $10 \mathrm{~min}$ and then drove manually for $10 \mathrm{~min}$. Then, the AD was available again and drivers tried to sleep again. If sleep stage N2 was confirmed a second time, drivers were awakened and the procedure with first the SICD and then the manual drive was triggered again. During both driving sessions, heart rate was measured with the Polar T34 chest belt as a measure for physiological activation.

Alertness is either measured with self-report measures such as a visual analogue scale or the Karolinska Sleepiness Scale, measures of cognitive performance- of which the PVT is arguably the most common—or physiologic measures [37]. All of them have a high inter-correlation and it is advised 
to use a combination of different measures. We therefore chose to use a combination of different alertness measures with the SAM-Scale as a self-report measure, the cardiac parameters as physiologic measures and the performance parameters of the SICD as measures of cognitive performance.

\subsection{Data Analysis}

For all indicators of arousal, repeated measures ANOVAs were conducted with the factors state and time. For state, three manifestations of the driver state were compared: after wakefulness (wakefulness, session 1), after drivers were asleep for the first time (after Sleep 1) and after drivers were asleep for the second time (after Sleep 2, both session 2). Furthermore, a factor time was analyzed which showed the change of the indicators over time.

For the subjective state, changes in arousal and well-being were compared for three points in time, directly after being awakened, after the SICD and after the manual drive. Mean and standard deviation of heart rate were analyzed as objective indicators for physiological arousal. Starting from the beginning of the SICD, parameters for heart rate were calculated for time segments of one minute duration, starting two minutes prior to the start of the SICD, including the $10 \mathrm{~min}$ of the SICD and $8 \mathrm{~min}$ of successive manual driving. In a similar approach, indicators measuring the performance in the SICD were calculated for segments with one minute of duration and analyzed over time. The proportion of hits and reaction times were calculated.

\section{Results}

\subsection{Subjective Arousal and Well-Being}

For the subjective arousal, there was a significant main effect of driver state $[\mathrm{F}(2,36)=9.898$, $p<0.000]$, a significant main effect of time $[\mathrm{F}(2,36)=17.069, p<0.000]$ and a significant interaction effect driver state*time $[\mathrm{F}(4,72)=6.499, p<0.000]$. Tukey post hoc test revealed that before the SICD (time point wake-up), the arousal after wakefulness was higher than after sleep. During the SICD, arousal increased for all three states. After the SICD, the differences between wakefulness and after sleep were no longer significant and were reduced further until after the drive. The development of the subjective arousal over time did not differ between after Sleep 1 and after Sleep 2.

For the subjective well-being, there was a main effect of driver state $[\mathrm{F}(2,36)=9.537, p<0.000]$, no effect of time $[\mathrm{F}(2,36)=0.159, p=0.853]$ and an interaction effect driver state time $[\mathrm{F}(4,7)=3.719$, $p=0.008]$. As can be seen in Figure 3, the only effect that could be interpreted is the interaction. After wake-up, subjective well-being was significantly lower after sleep than after wakefulness. Then, after sleep, there was a slight increase of well-being over time. On the contrary, subjective well-being decreased in the awake baseline condition. After the drive, subjective well-being after sleep and after wakefulness were on a similar level. Again, there was no difference in subjective state between Sleep 1 and Sleep 2.
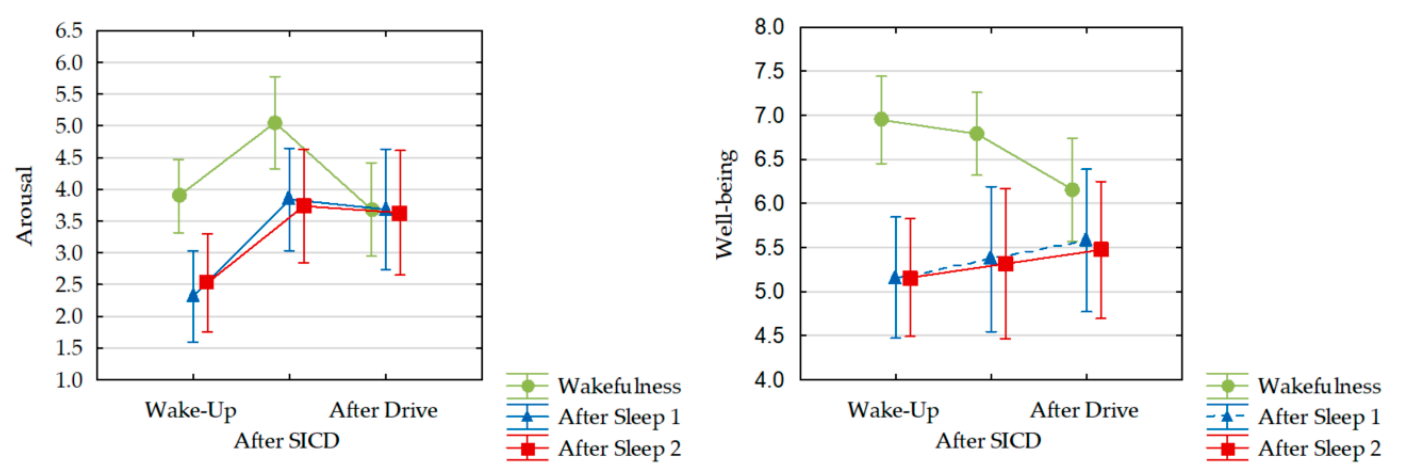

Figure 3. Subjective arousal (left) and well-being (right) after wake-up, after the SICD and after the manual drive for drivers after wakefulness and twice after sleep. The graph shows means and $95 \%$ confidence intervals. 


\subsection{Physiological Activation}

For the mean heartrate, there was a significant effect of time $[\mathrm{F}(19,361)=20.7, p<0.001]$ and a signficant interaction effect $[\mathrm{F}(38,722)=1.6177, p=0.012]$. For all states, there was an increase in heart rate with the beginning of the SICD which was more pronounced after sleep. During the SICD, the mean heart rate stayed on a constant level. After wakefulness, the increase of mean heart rate during the SICD was followed by a decrease during the manual drive. This decrease could not be found after sleep. For the standard deviation of heart rate, there was a significant effect of time $[\mathrm{F}(19,361)=31.22, p<0.001]$, of state $[\mathrm{F}(2,38)=4.42, p=0.019]$ and a significant interaction $[\mathrm{F}(38,722)$ $=10.505, p<0.000]$. All effects were based on a strong increase of heart rate variability during the process of waking up and starting with SICD after sleep. After wakefulness, heart rate variability stayed on a constant level throughout the analysed time frame. Means and standard deviations of heart rate during the SICD as well as during the manual drive are shown in Figure 4.
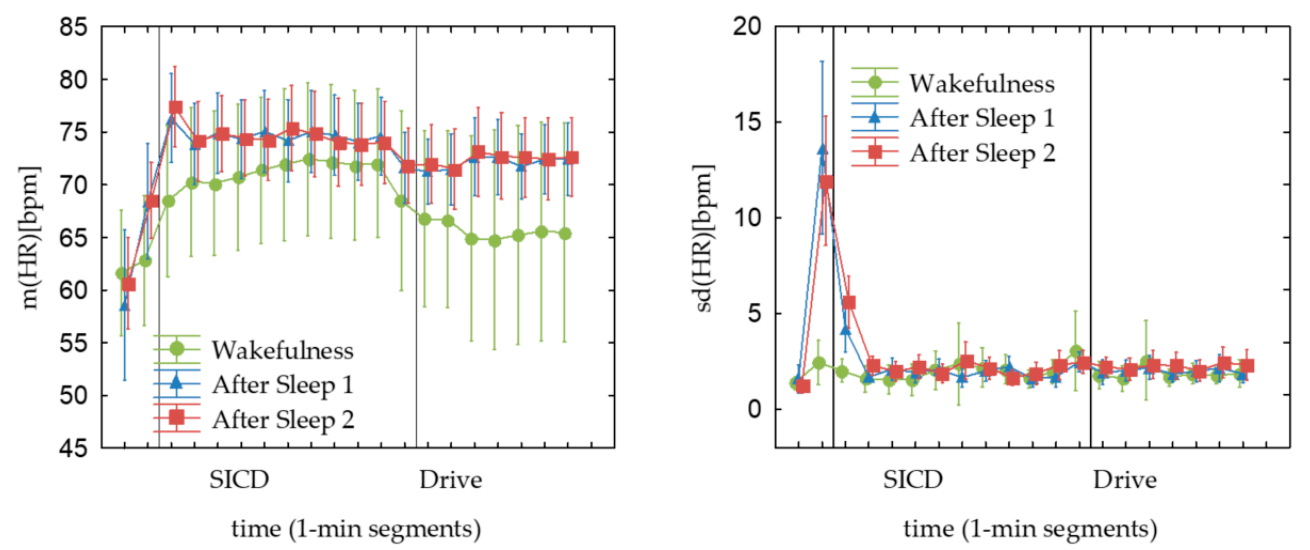

Figure 4. Mean (left) and standard deviation (right) of heart rate in time segments of 1 min before the SICD, during the SICD and during the successive manual drive.

Figure 5 shows an example of one driver's heart rate in the course of the drive. It illustrated how the heart rate was low during the automated drive due to a low arousal level of the driver. When the driver was awakened by the ADS, there was a sharp increase in heart rate and an overall higher level during the SICD and the manual drive. The arousal lowered as soon as the ADS was activated again.

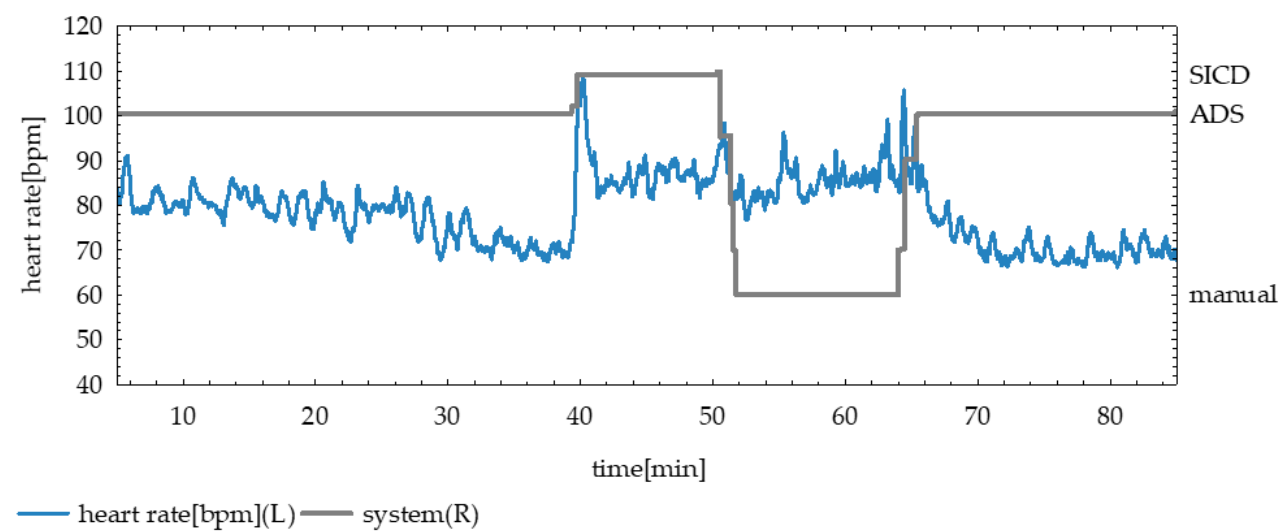

Figure 5. Example for change of heart rate for automated drive (ADS), during the SICD and during the manual drive and back to ADS. The driver was asleep during the automated drive (ADS).

\subsection{Subjective Evaluation of the SICD}

For assessing the acceptance of the SICD by users, the drivers were asked to rate the SICD on the 9-point acceptance scale after the drive in the second session. One sample $t$-tests were calculated 
against the scale mean (0). The SICD was perceived as assisting $(\mathrm{M}=-0.57, \mathrm{SD}=1.07, p=0.024)$ and marginally as good $(\mathrm{M}=0.38, \mathrm{SD}=0.8, p=0.057)$. All other scales did not differ from the scale mean. Drivers' acceptance of the SICD is depicted in Figure 6.

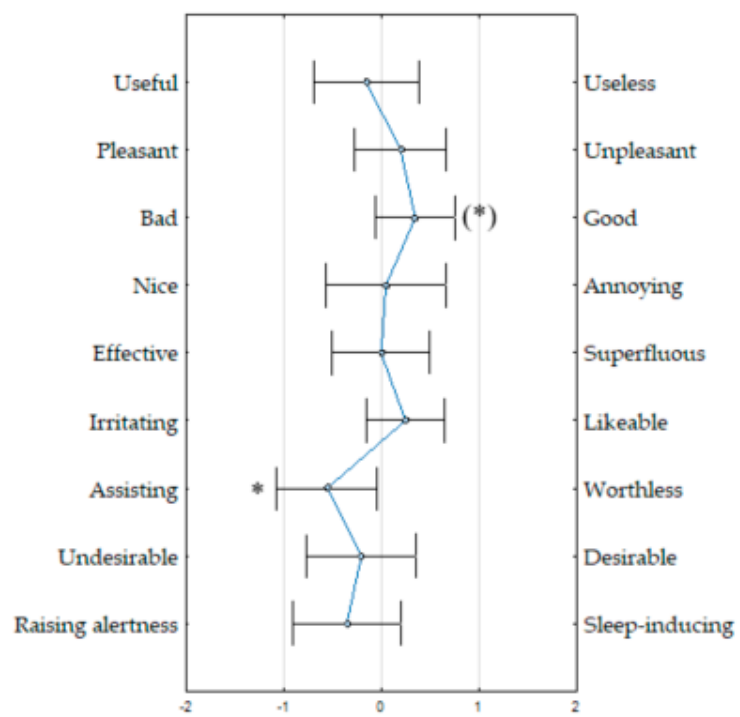

Figure 6. Means and 95\% confidence intervals for the acceptance of the SICD. Significant differences from the scale mean are marked with *, nearly significant differences with $\left(^{*}\right)$.

In the postquestionnaire, drivers were asked to judge their driving behaviour after sleep with an open question. Some answers revealed information about the evaluation of the SICD. Those answers were, e.g., "Directly at the beginning, I felt more awake, but this effect quickly changed after the game was finished", "When I first played the game, it was refreshing and activating, but when I had to play it again, it was rather sleep-inducing.", "The game was sleep-inducing, because it is too long and not varied enough", "At the beginning of the game, it is arousing and raises alertness, but after a while it becomes annoying and monotonous".

\subsection{Performance on the SICD}

For the parameters mean reaction time and standard deviation of reaction time there were no significant effects. For the proportion of hits there was a significant effect of time $[F(9,162)=2.1521$, $p=0.028]$, which was based on an increase during the first three minutes after the start of SICD. Figure 7 shows two of the performance parameters, as examples, of the SICD, the percentage of hits and the mean reaction time in the course of $10 \mathrm{~min}$.
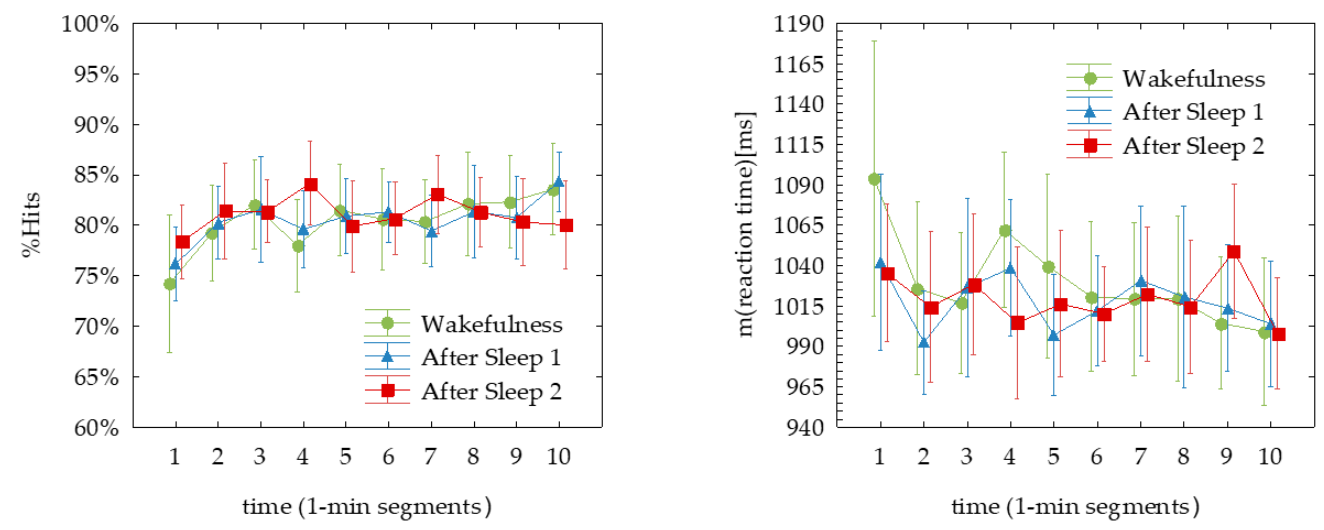

Figure 7. Proportion of hits of the SICD (left) and mean reaction times (right) for drivers after wakefulness, and twice after sleep. 


\section{Discussion}

Sleeping drivers in automated vehicles are already an issue today at a level of automation where the driver is clearly required to stay alert [30,31]. With the progression of automated driving technology and the development of dual-mode vehicles, sleep will arise as a use case and thus as a new driver state to be considered, e.g., in safety research and vehicle design. After sleep, human performance is impaired due to sleep inertia [6]. First studies on sleep in automated driving show that performance impairments are evident after awakening. There are more errors in take-over performance and manual driving performance is impaired $[10,11]$. The aim of the presented study was to develop a first implementation of a countermeasure to sleep inertia for drivers who are awakened during an automated drive. A sleep inertia counter-procedure for drivers (SICD) was developed. The purpose of the SICD was threefold: First, to activate the driver after awakening, second, to improve the driver's mood and motivation and third, to measure the driver's alertness.

The effectiveness and acceptance of the SICD was evaluated with $\mathrm{N}=21$ drivers who completed the SICD (a) after wakefulness and (b) after sleep. In both sessions we assessed physiological activation, subjective arousal and well-being, subjective evaluation of the SICD, as well as performance on the SICD.

\subsection{Activation of the Driver}

The SICD was designed especially to be activating. Drivers had to react quickly to achieve as many hits as possible and had to avoid distractor cues. Drivers report a higher subjective arousal after the SICD than before. This was the case after drivers had slept but also when they were awake. The activating effect of the SICD lasted until the end of the successive manual drive for drivers who had slept before but not for drivers who had been awake. The subjective arousal is also reflected by the physiological activation of the drivers. Drivers' activation, measured by the mean heart rate, is higher during the SICD than during the rather monotonous manual drive for drivers who had not slept. However, when drivers were awakened from sleep, the awakening process was highly activating and the heart rate was on a rather high level throughout the SICD but also during the manual drive. It seems that when drivers were asleep prior to the SICD, the SICD was able to physiologically activate the driver. This effect even lasted until after the SICD was finished and drivers drove manually. When drivers had not slept before, the SICD had a similar activating effect. However, after being awake, this effect did not last after the SICD was finished. The effects found for subjective arousal and for mean heart rate are similar: There was an increase of activation during the SICD which was followed again by a decrease during the manual drive after wakefulness. After sleep, the arousal level reached during SICD remained quite stable during the manual drive. Both the subjective and the objective activation effects after sleep were very stable and occurred in a similar way after both awakenings during the drive. Therefore, the aim of activating the driver with the SICD can be confirmed.

\subsection{Driver Mood and Attitude towards the SICD}

The drivers' subjective well-being was generally higher in the "awake" condition than after sleep which can be explained by the affective component of sleep inertia which is described as "grogginess". The SICD did not improve the drivers' well-being after sleep. The subjective evaluation of the SICD by drivers on the acceptance scale [38] was neutral. However, the drivers perceived the SICD as "assisting". The drivers stated that the SICD was too long and monotonous. The SICD could thus be improved by shortening the duration or by adding features that help to reduce monotony.

\subsection{Measuring Driver Alertness with the SICD}

One basic idea of the SICD was to use it as a diagnostic tool that reveals whether the driver's alertness has improved enough to consider her or him "ready to drive". Therefore, similar to classical measures of alertness like the PVT, performance parameters "hits", "misses", "fails" and "reaction 
times" were measured. Unfortunately, there is no effect of the drivers' state on the parameters of the SICD. In the current implementation it can therefore not be considered a valid diagnostic tool to assess drivers' readiness to drive. A simpler task is indicated where no learning effects can be expected. However, this goal might be challenging to be combined with the aim to implement a less monotonous task.

\subsection{Conclusions}

While the SICD proved to be subjectively and physiologically activating, the trade-off between motivating appeal and diagnostic capacity-similar to classic alertness tasks-turned out to be the essential challenge. Standardized and validated tasks like the PVT have the advantage that, due to the simplicity of the design, there are no learning effects and the subject's alertness can directly be derived from the performance parameters. However, this task could clearly not be considered motivating and rather, was annoying to the subject. We therefore tried to design our SICD such that it was more varied and added motivating messages. The drivers considered the SICD to be of assistance and the subjective arousal scales as well as the heart rate show that it was also activating. On the other hand, it was not capable of measuring alertness and thus its diagnostic properties could not be confirmed. The SICD was accepted by drivers and at least did not worsen the driver's well-being. On the other hand, driving behavior is impaired [11] and therefore, before handing the vehicle control over to the driver, some kind of performance check of the driver is indicated. The SICD did not reveal information about the driver's alertness. In summary, the SICD was able to fulfill two of the aims: to activate and to motivate the driver. The third aim, to measure the driver's alertness, was not accomplished.

\subsection{Limitations}

The main limitation for the interpretation of the results is the chosen study design. The two experimental conditions compared the full SICD in the state of sleep inertia to wakefulness as a baseline condition. However, to draw clear conclusions about the effectiveness of the SICD, the treatment (SICD) should be compared to a baseline (no SICD). The obtained physiological, subjective and performance data can only be evaluated in a timely perspective. The variance in the data might not only depend on the SICD but also on time effects and consequently a clear interpretation of the data is difficult. Future studies should directly compare different approaches to deal with sleep inertia. One approach could be to test the presented cognitive stimulation approach to the NASA nap paradigm, thus a reactive approach to a proactive approach. As other approaches, physical exercise or a combined physical-cognitive activation task (e.g., a cue-search task incorporating the whole vehicle cockpit) could be introduced. Despite the limitations posed by the study design, we can conclude that the approach of cognitive stimulation is a promising framework for activating the driver after sleep. However, direct comparison of different approaches is indicated to assess their effectiveness.

\subsection{Directions for Future Research}

The approach of cognitive stimulation proved to be effective to physiologically and subjectively activate the driver. However, the SICD was not capable of assessing the driver's readiness to drive. This could either be done by a sophisticated driver monitoring system that detects the driver state [37] or by a performance check as it was conceptualized in the SICD. The driver's performance capabilities are clearly reduced after sleep [9]. It is crucial for driving safety to detect the driver's readiness to drive [12]. If the driver is detected as being not ready to drive, appropriate actions have to be taken. When the driver of a dual-mode vehicle is awakened from sleep, the ADS has to ensure that the driver is cognitively alert to engage in vehicle control. The SICD was developed similar to the PVT, as a validated measure of alertness. The difference was only that it was conceptualized not as a single reaction task but as a choice-reaction task to make it more varied and therefore more appealing and motivating. Measuring alertness with cognitive tasks is an established approach, however, our task 
was not able to measure alertness reliably. Future task designs should be more similar to established tasks, e.g., simpler single-choice tasks where no learning effects can be expected.

Another promising approach is physiological activation instead or in combination with cognitive activation [39]. Cerebral blood flow is decreased in the sleep inertia period which delays the reinstatement of alertness. Physical exercises have the potential to increase the overall blood circulation and therefore counter physiological sleep inertia. The implementation of physical exercises in the vehicle cabin is restricted. However, it is imaginable to instruct the driver to do stretching exercises. Physiological activation on the other hand, does not ensure that vehicle control—a primarily cognitive task-can be safely executed. Therefore, a combination of physiological and cognitive stimulation seems promising. Future research is needed in order to compare the effectiveness of different SICD approaches and to develop a method that is capable of successfully activating the driver, of measuring alertness and is motivating at the same time.

It is clearly critical to establish a framework to avoid sleep inertia from becoming a safety issue in automated driving. In other operational areas, e.g., aviation, standardized guidelines are in place to avoid sleep inertia. In automated driving, there is no such framework. Our proposed approach of cognitive stimulation has the potential to activate the driver. However, a sleep inertia countermeasure can only be considered effective when the driver's alertness and thus readiness to drive can be determined reliably.

Author Contributions: Conceptualization, J.W., R.K.-M.; C.P., D.B., S.F. and A.P.; methodology, J.W. and R.K.-M.; software, S.F.; investigation, J.W. and R.K.-M.; resources, A.P.; data curation, R.K.-M., J.W. and B.M.; writing—original draft preparation, J.W.; writing—review and editing, B.M., C.P. and R.K.-M.; project management, C.P. and A.P.; project acquisition: C.P. and A.P.; funding acquisition, A.P. All authors have read and agreed to the published version of the manuscript.

Funding: This research was funded by Hyundai Motor Europe Technical Center GmbH.

Acknowledgments: The authors would like to thank the co-workers of the WIVW who supported the research team with providing a fruitful and creative research environment and with conceptualizing and building the driving simulator hardware and the SILAB driving simulation implementation used in the study: Alexandra Neukum, Mathias Gold, Michael Hanig, Stefan Ludwig, and Markus Tomzig.

Conflicts of Interest: The authors declare no conflict of interest.

\section{References}

1. Azmat, M.; Kummer, S.; Moura, L.T.; Gennaro, F.D.; Moser, R. Future Outlook of Highway Operations with Implementation of Innovative Technologies Like AV, CV, IoT and Big Data. Logistics 2019, 3, 15. [CrossRef]

2. Kyriakidis, M.; Happee, R.; de Winter, J.C. Public opinion on automated driving: Results of an international questionnaire among 5000 respondents. Transp. Res. Part F Traffic Psychol. Behav. 2015, 32, 127-140. [CrossRef]

3. Becker, T.; Herrmann, F.; Duwe, D.; Stegmüller, S.; Röckle, F.; Niko, U. Enabling the Value of Time; Fraunhofer Institute for Industrial Engineering IAO: Stuttgart, Germany, 2018; pp. 1-27.

4. Upender, R.P. Sleep Medicine, Public Policy, and Public Health. In Principles and Practices of Sleep Medicine; Kryger, M., Roth, T., Eds.; Elsevier: Philadelphia, PA, USA, 2017; Volume 6, pp. 638-645.

5. SAE. Taxonomy and Definitions for Terms Related to Driving Automation Systems for On-Road Motor Vehicles; SAE: Warrendale, PA, USA, 2018; Volume J3016.

6. Ferrara, M.; De Gennaro, L. The sleep inertia phenomenon during the sleep-wake transition: Theoretical and operational issues. Aviat. SpaceEnviron. Med. 2000, 71, 843-848.

7. Rosekind, M.R.; Smith, R.M.; Miller, D.L.; Co, E.L.; Gregory, K.B.; Webbon, L.L.; Gander, P.H.; Lebacqz, J.V. Alertness management: Strategic naps in operational settings. J. Sleep Res. 1995, 4, 62-66. [CrossRef]

8. EASA. Commission Regulation (EU) 965/2012 on air operations. Amendment 16. In Acceptable Means of Compliance (AMC) and Guidance Material (GM) to Annex IV: Commercial Air Transport Operations [Part-CAT]; EASA: Cologne, Germany, 2019.

9. CASA. Safety Behaviours. In Human Factors Resource Guide for Engineers; Civil Aviation Safety Authority: Canberra, Australia, 2013. 
10. Wörle, J.; Metz, B.; Othersen, I.; Baumann, M. Sleep in Highly Automated Driving: Take-over Performance after Waking Up. Accid. Anal. Prev. 2020, 144. [CrossRef]

11. Wörle, J.; Metz, B.; Baumann, M. Investigating sleep inertia in automated driving: Methodological considerations and results from a driving simulator study. Accid. Anal. Prev. 2020. Under review.

12. EuroNCAP. Euro NCAP 2025 Roadmap; EuroNCAP: Leuven, Belgium, 2017; pp. 1-17.

13. Tassi, P.; Muzet, A. Sleep inertia. Sleep Med. Rev. 2000, 4, 341-353. [CrossRef]

14. Carskadon, M.A.; Dement, W.C. Normal human sleep: An overview. In Principles and Practice of Sleep Medicine, 6th ed.; Kryger, M., Roth, T., Eds.; Elsevier: Philadelphia, PA, USA, 2017; Volume 4, pp. $15-24$.

15. AASM. The AASM Manual for the Scoring of Sleep and Associated Events: Rules, Terminology and Technical Specifications; American Academy of Sleep Medicine: Darien, IL, USA, 2017.

16. Balkin, T.J.; Braun, A.R.; Wesensten, N.J.; Jeffries, K.; Varga, M.; Baldwin, P.; Belenky, G.; Herscovitch, P. The process of awakening: A PET study of regional brain activity patterns mediating the re-establishment of alertness and consciousness. Brain 2002, 125, 2308-2319. [CrossRef]

17. Ferrara, M.; Curcio, G.; Fratello, F.; Moroni, F.; Marzano, C.; Pellicciari, M.C.; De Gennaro, L. The electroencephalographic substratum of the awakening. Behav. Brain Res. 2006, 167, 237-244. [CrossRef]

18. Marzano, C.; Ferrara, M.; Moroni, F.; De Gennaro, L. Electroencephalographic sleep inertia of the awakening brain. Neuroscience 2011, 176, 308-317. [CrossRef]

19. Hilditch, C.J.; McHill, A.W. Sleep inertia: Current insights. Nat. Sci. Sleep 2019, 11, 155-165. [CrossRef] [PubMed]

20. Van Dongen, H.P.; Price, N.J.; Mullington, J.M.; Szuba, M.P.; Kapoor, S.C.; Dinges, D.F. Caffeine eliminates psychomotor vigilance deficits from sleep inertia. Sleep 2001, 24, 813-819. [CrossRef] [PubMed]

21. Scheer, F.A.; Shea, T.J.; Hilton, M.F.; Shea, S.A. An endogenous circadian rhythm in sleep inertia results in greatest cognitive impairment upon awakening during the biological night. J. Biol. Rhythm. 2008, 23, 353-361. [CrossRef] [PubMed]

22. Hilditch, C.J.; Centofanti, S.A.; Dorrian, J.; Banks, S. A 30-minute, but not a 10-minute nighttime nap is associated with sleep inertia. Sleep 2016, 39, 675-685. [CrossRef]

23. Groeger, J.A.; Lo, J.C.; Burns, C.G.; Dijk, D.-J. Effects of sleep inertia after daytime naps vary with executive load and time of day. Behav. Neurosci. 2011, 125, 252. [CrossRef] [PubMed]

24. McHill, A.W.; Hull, J.T.; Cohen, D.A.; Wang, W.; Czeisler, C.A.; Klerman, E.B. Chronic sleep restriction greatly magnifies performance decrements immediately after awakening. Sleep 2019, 42, zsz032. [CrossRef]

25. Dinges, D.F.; Orne, M.T.; Orne, E.C. Assessing performance upon abrupt awakening from naps during quasi-continuous operations. Behav. Res. MethodsInstrum. Comput. 1985, 17, 37-45. [CrossRef]

26. Silva, E.J.; Duffy, J.F. Sleep inertia varies with circadian phase and sleep stage in older adults. Behav. Neurosci. 2008, 122, 928. [CrossRef]

27. Bruck, D.; Pisani, D.L. The effects of sleep inertia on decision-making performance. J. Sleep Res. 1999, 8, 95-103. [CrossRef]

28. Cavallero, C.; Versace, F. Stage at awakening, sleep inertia and performance. Sleep Res. Online 2003, 5, 89-97.

29. Hayashi, M.; Motoyoshi, N.; Hori, T. Recuperative power of a short daytime nap with or without stage 2 sleep. Sleep 2005, 28, 829-836.

30. Signal, T.L.; Gander, P.; van den Berg, M.; O’Keeffe, K. Magnitude and Time Course of Sleep Inertia; Sleep/Wake Research Center: Wellington, New Zealand, 2008.

31. Hilditch, C.J.; Dorrian, J.; Banks, S. Time to wake up: Reactive countermeasures to sleep inertia. Ind. Health 2016, 54, 528-541. [CrossRef] [PubMed]

32. Solon, O. Who's driving? Autonomous cars may be entering the most dangerous phase. Guardian 2018, 9, 2019.

33. Guarino, B. Man Appears to Snooze at the Wheel of His Tesla while the Car Drives Itself on L.A. Highway; The Washington Post: Washington, DC, USA, 2016.

34. Oh, K.T.; Shin, J.; Kim, J.; Ko, M. Analysis of a Wake-Up Task-Based Mobile Alarm App. Appl. Sci. 2020, 10, 3993. [CrossRef]

35. Bradley, M.M.; Lang, P.J. Measuring emotion: The self-assessment manikin and the semantic differential. J. Behav. Ther. Exp. Psychiatry 1994, 25, 49-59. [CrossRef]

36. Jasper, H. Report of the committee on methods of clinical examination in electroencephalography. Electroencephalogr. Clin. Neurophysiol. 1958, 10, 370-375. 
37. Gabehart, R.J.; van Dongen, H.P. Circadian Rhythms in Sleepiness, Alertness, and Performance. In Principles and Practice of Sleep Medicine; Kryger, M.H., Roth, T., Eds.; Elsevier: Philadelphia, PA, USA, 2017; Volume 5, pp. 388-394.

38. Van Der Laan, J.D.; Heino, A.; De Waard, D. A simple procedure for the assessment of acceptance of advanced transport telematics. Transp. Res. Part C Emerg. Technol. 1997, 5, 1-10. [CrossRef]

39. Kovac, K.; Ferguson, S.A.; Paterson, J.L.; Aisbett, B.; Hilditch, C.J.; Reynolds, A.C.; Vincent, G.E. Exercising caution upon waking-can exercise reduce sleep inertia? Front. Physiol. 2020, 11, 254. [CrossRef] [PubMed]

(C) 2020 by the authors. Licensee MDPI, Basel, Switzerland. This article is an open access article distributed under the terms and conditions of the Creative Commons Attribution (CC BY) license (http://creativecommons.org/licenses/by/4.0/). 